EESTI NSV TEADUSTE AKADEEMIA TOIMETISED. XV KOIDE

FUUSIKA-MATEMAATIKA. JA TEHNIKATEADUSTE SEERIA, 1966, NR. 4

ИЗВЕСТИЯ АКАДЕМИИ НАУК ЭСТОНСКОИ ССР. ТОМ ХV

СЕРИЯ ФИЗИҚО-МАТЕМАТИЧЕСҚИХ И ТЕХНИЧЕСКИХ НАУК. 1966, № 4

И. КЕИС

\title{
ОДНА ЗАДАЧА ЭНЕРГЕТИЧЕСКОЙ ОПТИМИЗАЦИИ ДВИЖЕНИЯ ОТНОСИТЕЛЬНО ЦЕНТРА ИНЕРЦИИ ГИРОСТАТИЧЕСКОГО УСТРОЙСТВА ПЕРЕМЕННОЙ МАССЫ
}

1. Представим себе гиростат $G$ с центром инерции в точке $O$, снабженный баллонами, содержащими газ, соответственно расположенными и с такой системой управления, что при истечении газа из трех сопел, лежащих в главных плоскостях инерции $G$ относительно точки $O$ и торцами перпендикулярно своим радиус-векторам, тензор инерции совокупного тела $H$ остается подобным начальному

$$
\|I(t)\|=\left[1-x_{4}(t)\right]\left\|I\left(t_{0}\right)\right\|,
$$

а центр инерции совокупного тела совпадает с точкой $O$, неподвижной в теле $G$.

Если первоначальная масса расходуемого газа $m$, гиростата $M$, то не убывающая функция времени $x_{4}$ заключена, очевидно, в пределах

$$
0 \leqslant x_{4} \leqslant \frac{m}{m+M} .
$$

Обозначая через $\xi_{1}, \xi_{2}, \xi_{3}$ мгновенные расходы масс, истекающих со скоростью $v$ через торцы сопел, и пренебрегая в рассмотрении действием ссответствуюших им сил инерции, получим для проекций моментов реактивных сил значения $\delta_{0} v \xi_{1}, \delta_{0} v \xi_{2}, \delta_{0} v \xi_{3}\left(\delta_{0}-\right.$ расстояние от торца до $O)$. Примем для $v, \xi_{i}$ и проекций момента относительного количества двужения $k_{i}$ ограничения

$$
|v| \leqslant v_{0}, \quad 0 \leqslant \xi_{i} \leqslant \xi_{0},\left|k_{i}\right| \leqslant k_{0} .
$$

Для простоты подчиним $x_{4}$ уравнению

$$
d x_{4} / d t=\chi_{0}\left(\xi_{1}+\xi_{2}+\xi_{3}\right),
$$

в котором $\chi_{0}-$ постоянный и положительный параметр системы $H$ Пусть поле внешних сил, действующих на $H$, допускает существование силовой функции $U=U\left(R, i_{1}, i_{2}, \sigma_{1}, \sigma_{2}, \sigma_{3}\right)$, где $i_{1}$ и $i_{2}$ - широта и долгота относительного расположения $O$ и $O^{\prime}$, а $\sigma_{1}, \sigma_{2}, \sigma_{3}$ - проекции на главные оси инерции $H$ единичного вектора $\bar{\sigma}$, задающего направление луча из $O$ в некоторую точку пространства $O^{\prime}$, а $R$ есть расстояние между $O$ и $O^{\prime}$.

Уравнения движения системы $H$ относительно $O$ согласно $\left[{ }^{1,2}\right]$ суть: 


$$
\begin{gathered}
I_{1} \frac{d p}{d t}+\left(I_{3}-I_{2}\right) q r+\frac{d k_{1}}{d t}+q k_{3}-r k_{2}=\delta_{0} v \xi_{1}+\sigma_{3} \frac{\partial}{\partial \sigma_{2}} U-\sigma_{2} \frac{\partial}{\partial \sigma_{3}} U \\
I_{2} \frac{d q}{d t}+\left(I_{1}-I_{3}\right) p r+\frac{d k_{2}}{d t}+r k_{1}-p k_{3}=\delta_{0} v \xi_{2}+\sigma_{1} \frac{\partial}{\partial \sigma_{3}} U-\sigma_{3} \frac{\partial}{\partial \sigma_{1}} U \\
I_{3} \frac{d r}{d t}+\left(I_{2}-I_{1}\right) p q+\frac{d k_{3}}{d t}+p k_{2}-q k_{1}=\delta_{0} v \xi_{3}+\sigma_{2} \frac{\partial}{\partial \sigma_{1}} U-\sigma_{1} \frac{\partial}{\partial \sigma_{2}} U \\
\sin \Theta d \psi / d t=p \sin \varphi+q \cos \varphi \\
d \varphi / d t=r-\operatorname{ctg} \Theta(p \sin \varphi+q \cos \varphi) \\
d \Theta / d t=p \cos \varphi-q \sin \varphi \\
\sigma_{1}=\sin \Theta \sin \varphi, \quad \sigma_{2}=\sin \Theta \cos \varphi, \quad \sigma_{3}=\cos \Theta
\end{gathered}
$$

в которых $v, \xi_{i}, k_{i}$ подчинены ограничениям (1.3), $\|I(t)\|$ - равенству (1.1), а величина $x_{4}$ из (1.2) - уравнению (1.4). Если, однако, допустить, что $O$ и $O^{\prime}$ не являются фиксированными точками неподвижного пространства $O_{1} \xi \eta \xi$, то систему уравнений (1.6) удобно заменить следующей совокупностью векторных уравнений:

$$
\begin{aligned}
& \frac{d \bar{\sigma}}{d t}=[\bar{\sigma}, \bar{\Omega}]+\frac{\bar{v}-\dot{R} \bar{\sigma}}{R} \\
& \frac{d \bar{\beta}}{d t}=[\bar{\beta}, \bar{\Omega}] \\
& \frac{d \bar{\gamma}}{d t}=[\bar{\gamma}, \bar{\Omega}] \\
& \bar{\Omega}=(p, q, r),
\end{aligned}
$$

в которой $\bar{v}$ - разность скоростей точек $O^{\prime}$ и $O$, а $\bar{\beta}$ и $\bar{\gamma}-$ фиксированные и ортогональные векторы пространства $O_{1} \xi \eta \xi(\bar{\gamma}$ направлен для удобства вычислений вдоль $\left.O_{1} \xi\right)$, причем всюду предполагается, что величины $R$ и $\dot{R}$, а также проекции $\vec{v}$ на $O_{1} \xi \eta \zeta-$ известные функции времени. В этом случае вектор-функции $\bar{\sigma}, \bar{\beta}, \bar{\gamma}$ определяют взаимную ориентацию систем $O x y z$ и $O_{1} \xi \eta \xi$.

Условимся рассматривать лишь такую совокупность движений центров $O$ и $O^{\prime}$, при которой

$$
(\bar{v}, \bar{\gamma})=0, \quad(\bar{\sigma}, \bar{\gamma})=0
$$

названную здесь орбитальной.

Тогда в рассмотрении задачи можем опустить систему уравнений (1.9), а систему уравнений (1.7) записать в виде (согласно (1.10)):

$$
\frac{d \bar{\sigma}}{d t}=[\bar{\sigma}, \bar{\Omega}]+\frac{(\bar{\gamma},[\bar{\sigma}, \bar{v}])}{R n_{1}}\left(\bar{\beta}-n_{2} \bar{\sigma}\right)
$$

где $n_{1}$ и $n_{2}$ - проекции $\bar{\sigma}$ на $[\bar{\beta}, \bar{\gamma}]$ и $\bar{\beta}$, предполагаемые известными функциями времени. 
Предположим для простоты, что сопло с мгновенным расходом массы $\xi_{1}$ параллельно $O y$, а сопла с расходами $\xi_{2}$ и $\xi_{3}$ параллельны соответственно осям $\mathrm{Oz}$ и $\mathrm{Ox}$, а выражение живой силы соответствующей подвижным в теле $G$ элементам есть

$$
\frac{h}{2}\left(k_{1}^{2}+k_{2}^{2}+k_{3}^{2}\right)
$$

где $h$ - некоторая постоянная.

Используя эти допущения, получим согласно $\left[{ }^{1,2}\right]$ значение работы внутренних сил, отсчитываемой от некоторого начального момента времени $t_{0}$, до конечного момента времени $t_{2}$ в виде

$$
\begin{aligned}
E_{(0)}^{(2)} & =E\left(t_{2}\right)-E\left(t_{0}\right)=\left[\frac{1}{2}\left(I_{1} \omega_{1}^{2}+I_{2} \omega_{2}^{2}-I_{3} \omega_{3}^{2}\right)+k_{1} p+k_{2} q+k_{3} r+\right. \\
+ & \left.\frac{h}{2}\left(k_{1}^{2}+k_{2}^{2}+k_{3}^{2}\right)-U\right]_{(0)}^{(2)}+\frac{1}{2} \int_{t_{0}}^{t_{2}}\left\{v^{2}\left(\xi_{1}+\xi_{2}+\xi_{3}\right)+\delta_{0}^{2}\left[\left(p^{2}+q^{2}\right) \xi_{1}+\right.\right. \\
& \left.\left.+\left(q^{2}+r^{2}\right) \xi_{2}+\left(p^{2}+r^{2}\right) \xi_{3}\right]-2 \delta_{0} v\left(p \xi_{1}+q \xi_{2}+r \xi_{3}\right)\right\} d t
\end{aligned}
$$

Подынтегральное выражение $2 f^{(0)}(\nu, \xi, \tau)$ в формуле $(1.12)$ определяет удвоенную мощность, потребную для выброса частиц газа, тогда как остальные члены имеют обычный динамический смысл.

Поставим теперь следующую задачу.

В момент времени $t_{0}$ взаимная ориентация систем Охуд и $O_{1} \xi \eta$ задана векторами $\bar{\sigma}\left(t_{0}\right)$ и $\bar{\beta}\left(t_{0}\right)$; система $H$ имеет угловую скорость вращения относительно $O$, определяемую вектором $\bar{\Omega}\left(t_{0}\right)$; в момент $t_{0}$ вектор $\vec{k}=0$. Начиная с момента $t_{1}, t_{0} \leqslant t_{1}<t_{2}$, система $H$ должна совершить «мягкий» маневр, который достижим либо при соблюдении условий (1.3), либо же критерия

$$
|v| \leqslant v_{0}, \quad 0 \leqslant \xi_{i} \leqslant \xi_{0},\left|\frac{d_{1} k_{i}}{d_{1} t}\right| \leqslant k_{10}
$$

при котором $\bar{\Omega}(t), \bar{\sigma}(t), \bar{\beta}(t)$ суть заданные вектор-функции времени для $t_{1} \leqslant t \leqslant t_{2}$, причем в конечный момент времени $t_{2}$ положено для простоты вычислений $\bar{\Omega}\left(t_{2}\right)=\bar{k}\left(t_{2}\right)=0$. Кроме того, предположим, что в течение маневра расходуется практически весь запас газа, то есть

$$
x_{4}\left(t_{2}\right)=m(m+M)^{-1}+\varepsilon x_{41}\left(t_{2}\right), \quad\|I(t)\|=\left\|I\left(t_{0}\right)\right\|+\varepsilon\left\|I_{1}(t)\right\| .
$$

Здесь индекс 1 соответствует абсолютной производной по времени в формуле (1.13), $\varepsilon$, как всюду в дальнейшем, обозначает достаточно малое для справедливости вычислений значение безразмерного параметра. Вопрос о корректности рассмотрения по первому приближению является самостоятельным и здесь не исследуется.

Потребуем теперь, чтобы описанное движение происходило при минимальном значении функционала $E$. Ввиду того, что движение системы $H$ распадается на два этапа, а также вследствие принципа оптимальности Беллмана и принятых предположений сформулированная задача распадается по методу решения на два этапа - на первом решается задача минимизации $E$ в пределах $t_{1}$ и $t_{2}$, когда $k_{i}$ и $x_{4}$ - коор- 
динаты, а $v$ и $\xi_{i}-$ управления, и второй этап, на котором минимизируется $E$ в пределах $t_{0}$ и $t_{1}$, причем $\bar{\Omega}, \bar{\sigma}$ - искомые вектор-функции, а $d_{1} k_{i} / d_{1} t-$ управления. Заметим, что случай $t_{0}=t_{1}$ требует согласованности начальных данных в начале движения и на маневре. Введем новое время $\tau$ и обозначения согласно формулам:

$$
\begin{aligned}
& t=t_{2}-\tau \\
& T=t_{2}-t_{1} \\
& E_{0}=\left(I_{10} \omega_{1}^{2}+I_{20} \omega_{2}^{2}+I_{30} \omega_{3}^{2}\right)_{T} \\
& \left(\frac{d w}{d \tau}\right)_{T}=w^{\prime} \\
& x_{i}=k_{i} \quad(i=\overline{1,3}) \\
& h_{i}=h \\
& (p, q, r)_{t \geqslant t_{1}}=\left(\omega_{1}, \omega_{2}, \omega_{3}\right) \\
& \varphi_{1}=\sigma_{2} \frac{\partial}{\partial \sigma_{3}} U-\sigma_{3} \frac{\partial}{\partial \sigma_{2}} U \\
& f_{1}=I_{10} \frac{d \omega_{1}}{d \tau}+\left(I_{20}-I_{30}\right) \omega_{2}, \omega_{3}
\end{aligned}
$$

(здесь $(1,2,3)$ - символ последовательной циклической замены индексов)

$$
x_{0}=-\frac{1}{2}\left[\left(1-x_{4}\right) E_{0}+2 \omega_{i} x_{i}+h_{i} x_{i}^{2}\right]+\int_{0}^{T} f^{(0)}(v, \xi, \tau) d \tau
$$

Согласно условиям задачи вопрос нахождения движений, осуществляющих минимум $E_{(1)}^{(2)}$, сохраняется для величины $x_{0}(T)$, что позволяет воспользоваться принципом максимума Л. Понтрягина [3]. Используя здесь обозначения (1.14), уравнения (1.4) и (1.5), следует решить систему уравнений

$$
\begin{gathered}
d x_{1} / d \tau=\omega_{2} x_{3}-\omega_{3} x_{2}-\delta_{0} v \xi_{1}+\left(x_{4}-1\right) f_{1}+\varphi_{1} \\
d x_{4} / d \tau=-\chi_{0}\left(\xi_{1}+\xi_{2}+\xi_{3}\right) \\
d \psi_{1} / d \tau=\omega_{2} \psi_{3}-\omega_{3} \psi_{2}+\left[h\left(\delta_{0} v \xi_{1}-\varphi_{1}\right)-\omega_{1}-h f_{1} x_{4}\right] \\
d \psi_{4} / d \tau={ }_{2} E_{0}^{\prime}-\left(f_{1} \omega_{1}+f_{2} \omega_{2}+f_{3} \omega_{3}\right)- \\
-h\left(f_{1} x_{1}+f_{2} x_{2}+f_{3} x_{3}\right)-\left(f_{1} \psi_{1}+f_{2} \psi_{2}+f_{3} \psi_{3}\right),
\end{gathered}
$$

в которой $v, \xi_{i}$ необходимо заменить на такие функции от $x, \psi, \tau$, при которых функция $K(\nu, \xi, x, \psi, \tau)$, в данном случае

$$
K=\lambda_{1}(v) \xi_{1}+\lambda_{2}(v) \xi_{2}+\lambda_{3}(v) \xi_{3},
$$




$$
\begin{aligned}
& \lambda_{1}=\lambda_{0}^{*}+\frac{\delta_{0}^{2}}{2} \omega_{3}^{2}-\delta_{0} v \mu_{1}-\frac{v^{2}}{2} \\
& \lambda_{2}=\lambda_{0}^{*}+\frac{\delta_{0}^{2}}{2} \omega_{1}^{2}-\delta_{0} v \mu_{2}-\frac{v^{2}}{2} \\
& \lambda_{3}=\lambda_{0}^{*}+\frac{\delta_{0}^{2}}{2} \omega_{2}^{2}-\delta_{0} v \mu_{3}-\frac{v^{2}}{2} \\
& \lambda_{0}^{*}=\frac{\chi_{0}}{2} E_{0}-\frac{\delta_{0}^{2}}{2}\left(\omega_{1}^{2}+\omega_{2}^{2}+\omega_{3}^{2}\right)-\chi_{0} \psi_{4} \\
& \mu_{i}=h x_{i}+\psi_{i} \quad(i=\overline{1,3})
\end{aligned}
$$

достигает максимума при ограничениях типа (1.3) или (1.13). (В дальнейшем предполагается, что $x_{i}$ не могут быть кусочно-постоянными).

Относительно граничных условий для функций $x, \psi$ в этой задаче известно, что

$x_{i}(0)=0, \quad x_{4}(0)=\frac{m}{m+M}, \quad \psi_{i}(T)=0, \quad \psi_{4}(T)=0 \quad(i=\overline{1,3})$.

Опустим в рассмотрении задачи особый класс движений, при которых хотя бы одно из $\lambda_{i}$ обращается в нуль на некотором интервале времени $\tau_{i}$, принадлежашем отрезку $[0, T]$.

Тогда $\xi_{i}$ - релейные управления, определяемые формулой

$$
\xi_{i}=\xi_{0} \operatorname{Sg} \lambda_{i}
$$

Здесь $\operatorname{Sg}(x)=1$ при $x>0$ и $\operatorname{Sg}(x)=0$ при $x<0$, а $v$ задается одной из двух формул согласно равенству:

$$
v=\left\{\begin{array}{r}
-\beta \text { для } \tau \text {, при которых }|\beta| \leqslant v_{0} \\
-v_{0} \operatorname{sign} \beta \text { для } \tau, \text { при которых }|\beta|>v_{0},
\end{array}\right.
$$

где $\beta=3^{-1} \delta_{0}\left[\mu_{1} \operatorname{Sg} \lambda_{1}+\mu_{2} \operatorname{Sg} \lambda_{2}+\mu_{3} \operatorname{Sg} \lambda_{3}\right]$.

Из формул $(1.20),(1.21)$ следует, что значения $v\left(\mu_{1}, \mu_{2}, \mu_{3}, \tau\right)$, вообще говоря, удовлетворяют одной из трех следующих возможностей

$$
\begin{aligned}
& v<0 \\
& v=0 \\
& v>0
\end{aligned}
$$

причем в случае (1.23) для каждого момента времени существует «пассивная» плоскость значений $\mu_{i}$, проходящая через начало координат:

$$
\mu_{1} \operatorname{Sg}\left(\lambda_{0}^{*}+\frac{\delta_{0}^{2}}{2} \omega_{3}^{2}\right)+\mu_{2} \operatorname{Sg}\left(\lambda_{0}^{*}+\frac{\delta_{0}^{2}}{2} \omega_{1}^{2}\right)+\mu_{3} \operatorname{Sg}\left(\lambda_{0}^{*}+\frac{\delta_{0}^{2}}{2} \omega_{2}^{2}\right)=0
$$


Для всех $\mu$, не лежащих на пассивной плоскости, рассмотрим функции

$\vartheta_{1}(v)=\frac{\lambda_{0}^{*}-\frac{v^{2}}{2}+\frac{\delta_{0}^{2}}{2} \omega_{3}^{2}}{\delta_{0} v}, \quad \vartheta_{2}(v)=\frac{\lambda_{0}^{*}-\frac{v^{2}}{2}+\frac{\delta_{0}^{2}}{2} \omega_{1}^{2}}{\delta_{0} v}, \vartheta_{3}(v)=\frac{\lambda_{0}^{*}-\frac{v^{2}}{2}+\frac{\delta_{0}^{2}}{2} \omega_{2}^{2}}{\delta_{0} v}$

и разности

$$
\mathrm{Q}_{i}=\vartheta_{i}(v)-\mu_{i} \quad(i=\overline{1,3})
$$

Случаи, когда совместно выполняются условия $(1.20)$ и (1.22), (1.20) и $(1.24),(1.21)$ и $(1.22),(1.21)$ и $(1.24)$ назовем соответственно случаями $A, B, C, D$. Изучим сперва случаи $A$. Для них, очевидно, справедливы равенства $\mathrm{Sg} \lambda_{i}=-\mathrm{Sg} \varrho_{i}$ (для случаев $B$ верно $\mathrm{Sg} \lambda_{i}=\operatorname{Sg} \varrho_{i}$ ). Случаи $A$ представимы восемью вариантами следующего вида:
1) $\mu_{1}>\vartheta_{1}, \quad \mu_{2}>\vartheta_{2}, \quad \mu_{3}>\vartheta_{3}, \quad 0<\mu_{1}+\mu_{2}+\mu_{3} \leqslant \chi_{0}$
2) $\mu_{1}<\vartheta_{1}, \quad \mu_{2}>\vartheta_{2}, \quad \mu_{3}>\vartheta_{3}, \quad 0<\mu_{2}+\mu_{3} \leqslant \chi_{0}$
3) $\mu_{1}<\vartheta_{1}, \quad \mu_{2}<\vartheta_{2}, \quad \mu_{3}>\vartheta_{3}, \quad 0<\mu_{3} \leqslant \chi_{0}$
4) $\mu_{1}>\vartheta_{1}, \quad \mu_{2}<\vartheta_{2}, \quad \mu_{3}>\vartheta_{3}, \quad 0<\mu_{1}+\mu_{3} \leqslant \chi_{0}$
5) $\mu_{1}>\vartheta_{1}, \quad \mu_{2}>\vartheta_{2}, \quad \mu_{3}<\vartheta_{3}, \quad 0<\mu_{1}+\mu_{2} \leqslant \chi_{0}$
6) $\mu_{1}<\vartheta_{1}, \quad \mu_{2}>\vartheta_{2}, \quad \mu_{3}<\vartheta_{3}, \quad 0<\mu_{2} \leqslant \chi_{0}$
7) $\mu_{1}<\vartheta_{1}, \quad \mu_{2}<\vartheta_{2}, \quad \mu_{3}<\vartheta_{3}$,
8) $\mu_{1}>\vartheta_{1}, \quad \mu_{2}<\vartheta_{2}, \quad \mu_{3}<\vartheta_{3}, \quad 0<\mu_{1} \leqslant \chi_{0}$,

$$
\text { rдe } \chi_{0}=3 \delta_{0}^{-1} v_{0} \text {. }
$$

Множество $A_{1}$ в пространстве $\mu$ отвечает условиям 1) и удовлетворяет, как нетрудно показать, неравенствам

$$
\begin{gathered}
\left(\mu_{1}+\mu_{2}+\mu_{3}\right)\left[5 \mu_{1}-\left(\mu_{2}+\mu_{3}\right)\right]>a_{11}=-9\left(\frac{2 \lambda_{0}^{*}}{\delta_{0}^{2}}+\omega_{3}^{2}\right) \\
\left(\mu_{1}+\mu_{2}+\mu_{3}\right)\left[5 \mu_{2}-\left(\mu_{1}+\mu_{3}\right)\right]>a_{12}=-9\left(\frac{2 \lambda_{0}^{*}}{\delta_{0}^{2}}+\omega_{1}^{2}\right) \\
\left(\mu_{1}+\mu_{2}+\mu_{3}\right)\left[5 \mu_{3}-\left(\mu_{1}+\mu_{2}\right)\right]>a_{13}=-9\left(\frac{2 \lambda_{0}^{*}}{\delta_{0}^{2}}+\omega_{2}^{2}\right) \\
0<\mu_{1}+\mu_{2}+\mu_{3} \leqslant \chi_{0} .
\end{gathered}
$$

Если заменить первые три неравенства на равенства, то легко заметить, что соответствующие им в пространстве $\mu$ поверхности $A_{1 i}$ представят собою гиперболические цилиндры с центром в $O$ и асимптотическими плоскостями

$\mu_{1}+\mu_{2}+\mu_{3}=5 \mu_{1}-\left(\mu_{2}+\mu_{3}\right)=5 \mu_{2}-\left(\mu_{1}+\mu_{3}\right)=5 \mu_{3}-\left(\mu_{1}+\mu_{2}\right)=0$. 
Числа $\lambda_{1}=-3, \lambda_{2}=0, \lambda_{3}=6$ являются собственными значениями оператсров $\left\|A_{1 i}\right\|$, так что для каждой поверхности $A_{1 i}$ существует единственное главное направление, пересекающее поверхности $A_{1 i}$ на расстоянии $a_{1 i}^{0}=\left(a_{1 i}\right)^{\frac{1}{2}} \lambda_{k}^{-\frac{1}{2}}(k-$ одно из $\overline{1,3)}$ от центра $O$ в $\mu$. Поверхности $A_{1 i}$ имеют образующие параллельно главным направлениям $\zeta_{1 i}$, отвечающим числу $\lambda=0$. Легко видеть в плоскости двух других главных направлений для каждой из поверхностей $A_{1 i}$, что существует не пустое пересечение внешней по отношению к гиперболе $g_{1 i}$ (след $A_{1 i}$ в плоскости $\left.\xi_{i}=0\right)$ и полосы $0<\sqrt{2} \xi_{1 i}-\eta_{1 i} \leqslant \chi_{0}$, определяюшей множество $(1.26)$, записанное в собственных координатах операторов $\left\|A_{1 i}\right\|$; последнее представляет собой гиперболический сегмент $\sigma_{1 i}$, тогда как в пространстве ему отвечает столб $\Gamma_{1 i}$ с образующей $\xi_{i}$ и основанием $\sigma_{1 i}$. Распределение главных осей $\xi_{1 i}, \eta_{1 i}, \xi_{1 i}$ в $\mu$ можно задать матрицей

\begin{tabular}{|c|c|c|c|c|c|c|c|}
\hline$\xi_{11}$ & $\eta_{11}$ & $\zeta_{11}$, & $\xi_{12}$ & $\eta_{12}$ & $\zeta_{12}$ & $\xi_{13}$ & $\eta_{13}$ \\
\hline$\frac{4}{3 \sqrt{2}}$ & $\frac{1}{3}$, & 0 , & $\frac{1}{3 \sqrt{2}}$ & $\frac{2}{3}$, & $\frac{1}{\sqrt{2}}$ & $\frac{1}{3 \sqrt{2}}$ & $\frac{2}{3}$, \\
\hline$\frac{1}{3 \sqrt{2}}$ & $-\frac{2}{3}$ & $\frac{1}{\sqrt{2}}$ & $\frac{4}{3 \sqrt{2}}$ & $-\frac{1}{3}$ & 0 & $\frac{1}{3 \sqrt{2}}$ & $\frac{2}{3}$, \\
\hline$\frac{1}{3 \sqrt{2}}$ & $-\frac{2}{3}$ & $-\frac{1}{\sqrt{2}}$ & $\frac{1}{3 \sqrt{2}}$ & $\frac{2}{3}$ & $-\frac{1}{\sqrt{2}}$ & $\frac{4}{3 \sqrt{2}}$ & $-\frac{1}{3}$ \\
\hline
\end{tabular}

Как следовало ожидать из выражений $a_{1 i}$, системы координат $O \xi_{13} \eta_{13} \zeta_{13}$, $O \xi_{12} \eta_{12} \xi_{12}, O \xi_{11} \eta_{11} \xi_{11}$ переходят одна в другую последовательными поворотами на угол $2^{-1} \pi$ относительно осей $O \eta_{11}, O \eta_{13}, O \eta_{12}$. Окончательно, пересечение столбов $\Gamma_{1 i}$ определяет в $\mu$ область $h_{1}^{(a)}$, в которой $v=-3^{-1} \delta_{0}\left(\mu_{1}+\mu_{2}+\mu_{3}\right)$. Множество $A_{2}$, согласно 2), задается неравенствами

$$
\begin{gathered}
\left(\mu_{2}+\mu_{3}\right)\left[6 \mu_{1}-\left(\mu_{2}+\mu_{3}\right)\right]<a_{21}=a_{11} \\
\left(\mu_{2}+\mu_{3}\right)\left(5 \mu_{2}-\mu_{3}\right)>a_{22}=a_{12} \\
\left(\mu_{2}+\mu_{3}\right)\left(5 \mu_{3}-\mu_{2}\right)>a_{23}=a_{13} \\
0<\mu_{2}+\mu_{3} \leqslant \chi_{0} .
\end{gathered}
$$

Вполне аналогично предыдущему получим для поверхностей $A_{2 i}$ асимптотические плоскости $\mu_{2}+\mu_{3}=6 \mu_{1}-\left(\mu_{2}+\mu_{3}\right)=5 \mu_{2}-\mu_{3}=5 \mu_{3}-\mu_{2}=$ $=0$. Поверхности $A_{22}$ и $A_{23}$ представляют собой гиперболические цилиндры с образующими параллельными оси $O \mu_{1}$, их $g_{2 i}(i=2,3)$ имеют обшую асимптоту $\mu_{1}=\mu_{2}+\mu_{3}=0$; в пересечении внешних по отношению $\mathrm{k} g_{2 i}$ частей плоскости $\mu_{1}=0$ получаем некоторое множество $\sigma_{223}$, которому в пространстве $\mu$ отвечает некоторый столб $\Gamma_{223}$; пересечение псследнего с внутренней частью пространства, ограниченного поверхностью $A_{21}$ и со слоем $(1.27)$, определяет в $\mu$ область $h_{2}^{(a)}$, в которой $v=-\left(\mu_{2}+\mu_{3}\right) \delta_{0} 3^{-1}$.

Для $A_{3}$, согласно 3 ), получаем неравенства:

$$
\begin{gathered}
\mu_{3}\left(6 \mu_{1}-\mu_{3}\right)<a_{31}=a_{11} \\
\mu_{3}\left(6 \mu_{2}-\mu_{3}\right)<a_{32}=a_{12} \\
5 \mu_{3}^{2}>a_{33}=a_{13}
\end{gathered}
$$




$$
0<\mu_{3} \leqslant \chi_{0}
$$

Для совместности неравенств (1.28) и (1.29) при $a_{13}>0$ необходимо, чтобы $\left(5^{-1} a_{13}\right)^{\frac{1}{2}} \leqslant \chi_{0}$, а $\mu_{3}$ принадлежало полуинтервалу $\sigma_{30}$ : $\left(\left(5^{-1} a_{13}\right)^{1 / 2}, \chi_{0}\right]$. Гиперболический цилиндр $A_{31}$ с осью, параллельной направлению $O \mu_{1}$, имеет асимптотические плоскости $\mu_{3}=0$ и $6 \mu_{1}-\mu_{3}=0$, тогда как гиперболический цилиндр $A_{32}$ с осью, параллельной направлению $O \mu_{2}$, имеет асимптотические плоскости $\mu_{3}=0$ и $6 \mu_{2}-\mu_{3}=0$. Пересечение соответствующих им внутренних областей пространства $\mu$ и слоя над $\sigma_{30}$ дает область $h_{3}^{(a)}$, в которой $v=-3^{-1} \delta_{0} \mu_{3}$. Заметим, что неравенства, определяюшие множество $A_{4}$ -

$$
\begin{gathered}
\left(\mu_{1}+\mu_{3}\right)\left[6 \mu_{2}-\left(\mu_{1}+\mu_{3}\right)\right]<a_{42}=a_{12} \\
\left(\mu_{1}+\mu_{3}\right)\left(5 \mu_{1}-\mu_{3}\right)>a_{41}=a_{11} \\
\left(\mu_{1}+\mu_{3}\right)\left(5 \mu_{3}-\mu_{1}\right)>a_{43}=a_{13} \\
0<\mu_{1}+\mu_{3} \leqslant \chi_{0} \\
3 v=-\delta_{0}\left(\mu_{1}+\mu_{3}\right)
\end{gathered}
$$

могут быть получены из указанных для множества $A_{2}$, если в последних сделать подстановку $1-2$ :

(2) 1 )

так что множество $h_{4}^{(a)}$ получается из множества $h_{2}^{(a)}$ поворотом последнего в пространстве $\mu$ на некоторый угол вокруг оси $O \mu_{3}$ и преобразованием подобия. Аналогично множество $h_{5}^{(a)}$ согласно неравенствам

$$
\begin{gathered}
\left(\mu_{1}+\mu_{2}\right)\left(5 \mu_{1}-\mu_{2}\right)>a_{51} \\
\left(\mu_{1}+\mu_{2}\right)\left(5 \mu_{2}-\mu_{1}\right)>a_{52} \\
\left(\mu_{1}+\mu_{2}\right)\left[6 \mu_{3}-\left(\mu_{1}+\mu_{2}\right)\right]<a_{53} \\
0<\mu_{1}+\mu_{2} \leqslant \chi_{0} \\
3 v=-\delta_{0}\left(\mu_{1}+\mu_{2}\right)
\end{gathered}
$$

может быть получено из $h_{2}^{(a)}$ поворотом последнего относительно оси $O \mu_{2}$ и преобразованием подобия. Таким же путем множество $h_{6}^{(a)}$

$$
\begin{gathered}
\mu_{2}\left(6 \mu_{1}-\mu_{2}\right)<a_{61} \\
\mu_{2}\left(6 \mu_{3}-\mu_{2}\right)<a_{63} \\
5 \mu_{2}^{2}>a_{62} \\
0<\mu_{2} \leqslant x_{0}, \quad\left(3 v_{0}=-80 \mu_{2}\right)
\end{gathered}
$$

получается из $h_{3}^{(a)}$ поворотом относительно $O \mu_{1}$ и преобразованием подобия. 
Согласно предположению о $\lambda_{i}$ множесгво $A_{7}$ продолжает пассивную плоскость, а множество $h_{8}^{(a)}$ получается из $h_{6}^{(a)}$ поворотом вокруг $O \mu_{3}$ и пресбразованием подобия, причем $v=-3^{-1} \delta_{0} \mu_{1}$.

Нетрудно убедиться далее, что между множествами $h_{j}^{(a)}$ и $h_{k}^{(b)}$, соотгетствующими $A$ и $B$, можно установить соответствие, при котором внутри соответствующих областей $v$ определяется одной и той же аналитической функцией от $\mu_{1}, \mu_{2}, \mu_{3}$, а сами области симметричны относительно начала координат. Например, $B_{3}$ и $A_{5}$. Тогда относительно множеств $A$ и $B$ можно сказать, что в построенной системе областей $h_{j}^{(a)}$, $h_{k}^{(b)} v-$-известная функция от $\mu_{1}, \mu_{2}, \mu_{3} ;$ удаление каждой из этих областей от центра есть функция модулей величин $a_{11}, a_{12}, a_{13}$, знак которых определяет сриентацию относительно главных осей $\xi_{k i}, \eta_{k i}$, $\xi_{k i}$ и некоторых точек на ссях $O \mu_{1}, O \mu_{2}, O \mu_{3}$ этих областей.

Рассмотрим случаи $C$, для которых

$$
v=-v_{0} \text {. }
$$

Согласно формулам (1.21) и (1.22) имеем для $C$ неравенства

$$
\begin{array}{llll}
C_{1}: \mu_{1}>-\varepsilon_{1}, & \mu_{2}>-\varepsilon_{2}, & \mu_{3}>-\varepsilon_{3}, & \mu_{1}+\mu_{2}+\mu_{3}>\chi_{0} \\
C_{2}: \mu_{1}<-\varepsilon_{1}, & \mu_{2}>-\varepsilon_{2}, & \mu_{3}>-\varepsilon_{3}, & \mu_{2}+\mu_{3}>\chi_{0} \\
C_{3}: \mu_{1}<-\varepsilon_{1}, & \mu_{2}<-\varepsilon_{2}, & \mu_{3}>-\varepsilon_{3}, & \mu_{3}>\chi_{0} \\
C_{4}: \mu_{1}>-\varepsilon_{1}, & \mu_{2}<-\varepsilon_{2}, & \mu_{3}>-\varepsilon_{3}, & \mu_{1}+\mu_{3}>\chi_{0} \\
C_{5}: \mu_{1}>-\varepsilon_{1}, & \mu_{2}>-\varepsilon_{2}, & \mu_{3}<-\varepsilon_{3}, & \mu_{1}+\mu_{2}>\chi_{0} \\
C_{6}: \mu_{1}<-\varepsilon_{1}, & \mu_{2}>-\varepsilon_{2}, & \mu_{3}<-\varepsilon_{3}, & \mu_{2}>\chi_{0} \\
C_{7}: \mu_{1}>-\varepsilon_{1}, & \mu_{2}<-\varepsilon_{2}, & \mu_{3}<-\varepsilon_{3}, & \mu_{1}>\chi_{0},
\end{array}
$$

каждой строке которых соответствует в пространстве $\mu$ область $h_{i}^{(c)}$. Для $D$ аналогичным образом можно получить области $h_{j}^{(d)}$, симметричные соответствующим областям $h_{i}^{(c)}$ относительно начала $O$. Қ примеру, для $D_{1}$ имеем строку $\mu_{1}<\varepsilon, \mu_{2}>\varepsilon_{2}, \mu_{3}>\varepsilon_{3}, \mu_{1}<-\chi_{0}$. Здесь введены обозначения

$$
\varepsilon_{1}=\frac{\lambda_{0}^{*}+\frac{\delta_{j}^{2}}{2} \omega_{3}^{2}-\frac{v_{0}^{2}}{2}}{\delta_{0} v_{0}}, \quad \varepsilon_{2}=\frac{\lambda_{0}^{*}+\frac{\delta_{0}^{2}}{2} \omega_{1}^{2}-\frac{v_{0}^{2}}{2}}{\delta_{0} v_{0}}, \quad \varepsilon_{3}=\frac{\lambda_{0}^{*}+\frac{\delta_{0}^{2}}{2} \omega_{2}^{2}-\frac{v_{0}^{2}}{2}}{\delta_{0} v_{0}}
$$

2. Поскольку в дальнейшем рассматриваются орбитальные движения, для которых $\left(R n_{1}\right)^{-1}(\bar{\gamma},[\bar{\sigma}, \bar{v}]) \simeq \varepsilon$, то уравнения $(1.8),(1.9)$ и $(1.11)$ образуют систему уравнений, эквивалентную системе уравнений Пуассона, то есть решение последней легко указать, как только известны $\bar{\sigma}$, $\bar{\beta}, n_{1}, n_{2}$. В течение маневра эти функции известны по условию задачи, а вместе с ними - решение системы уравнений Пуассона, два первых векторных уравнения которой записываются для $\tau$ в виде однородных уравнений, ссответствующих $\varkappa_{i}$ и $\psi_{i}$ из (1.15). Обозначим известную такім образом матрицу решений однородной системы уравнений (1.15) $\varkappa_{i}^{j}$. Смысл этой матрицы определяется следующим образом: 


$$
a_{i}^{j}=\left\|\alpha_{i}^{j}(\tau)\right\|=\left(\begin{array}{l}
\alpha_{1} \beta_{1} \gamma_{1} \\
\alpha_{2} \beta_{2} \gamma_{2} \\
\alpha_{3} \beta_{3} \gamma_{3}
\end{array}\right)
$$

где взаимно ортогональные и нормированные столбцы представляют собою решение уравнений Пуассона.

Используя метод вариации постоянных Лагранжа, а также составив комбинации

$$
\begin{gathered}
\zeta_{0}=2^{-1} E_{0}^{\prime}-\left(f_{1} \omega_{1}+f_{2} \omega_{2}+f_{3} \omega_{3}\right), \quad \zeta_{i}=-\left(\omega_{i}^{\prime}+h \varphi_{i}\right), \\
S_{i}=\varphi_{i}-f_{i} \quad(i=\overline{1,3}),
\end{gathered}
$$

получим из (1.15) с учетом (1.14) (1.19) и (2.1) уравнения для $y_{i}$ и $z_{v}\left(x_{i}=\alpha_{i}^{i} y_{i}, \psi_{k}=\alpha_{k}^{\nu} z_{v}\right)$

$$
\begin{aligned}
a_{i}^{j} \frac{d y_{j}}{d \tau} & =S_{i}+f_{i} x_{4}-\delta_{0} \xi_{0} \nu \operatorname{Sg} \lambda_{i} \\
a_{i}^{j} \frac{d z_{j}}{d \tau} & =\xi_{i}-h f_{i} x_{4}+h \delta_{0} \xi_{0} \nu \operatorname{Sg} \lambda_{i} \\
\frac{d x_{4}}{d \tau} & =-\chi_{0} \xi_{0}\left(\operatorname{Sg} \lambda_{1}+\operatorname{Sg} \lambda_{2}+\operatorname{Sg} \lambda_{3}\right) \\
\frac{d \psi_{4}}{d \tau} & =\zeta_{0}-\left(f_{1} \mu_{1}+f_{2} \mu_{2}+f_{3} \mu_{3}\right)
\end{aligned}
$$

Определим комплексы $\mho_{\nu}(\tau)$ равенствами $\mu_{i}=\alpha_{i}^{v} w_{\nu}$; для них из (2.2) и (2.3) получим уравнения

$$
\frac{d w_{j}}{d \tau}=\alpha_{j}^{i}\left(\zeta_{i}+h S_{i}\right)
$$

которые имеют решения

$$
w_{j}(\tau)=w_{j}(0)+\int_{0}^{\tau} a_{j}^{i}\left(\zeta_{i}+h S_{i}\right) d \tau
$$

Следовательно, комплексы $\mu_{i}(\tau)$ определяются равенствами

$$
\mu_{i}(\tau)=\alpha_{i}^{v}(\tau)\left\{w_{\nu}(0)+\int_{0}^{\xi} a_{v}^{k}(\xi)\left[\zeta_{k}(\xi)+h S_{k}(\xi)\right] d \xi\right\}
$$

Импульс $\psi_{4}$ согласно (2.5), а также условию (1.18) есть функция

$$
\psi_{4}(\tau)=\int_{0}^{T}\left(f_{1} \mu_{1}+f_{2} \mu_{2}+f_{3} \mu_{3}-\zeta_{0}\right) d \tau+\int_{0}^{\tau}\left[\xi_{0}-\left(f_{1} \mu_{1}+f_{2} \mu_{2}+f_{3} \mu_{3}\right)\right] d \tau
$$

в выражении которой комплексы $\mu_{i}$ необходимо заменить из равенств (2.7). 
Координата $x_{4}$, ввиду уравнения (2.4) и условия (1.18), есть следующая функция $\tau$ :

$$
x_{4}=\frac{m}{m+M}-\chi_{0} \xi_{0}\left[\tau_{1}(\tau)+\tau_{2}(\dot{\tau})+\tau_{3}(\tau)\right] .
$$

Здесь $\tau_{i}(\tau)$ - интервал времени, отвечающий положительным значениям $\lambda_{l}$.

Ввиду условий (1.18) и уравнений $(2.2)$ имеем для $y_{i}$ и $x_{i}$ формулы

$$
\begin{gathered}
y_{j}(\tau)=\int_{0}^{\tau} a_{j}^{i}\left(S_{i}+f_{i} x_{4}-\delta_{0} \xi_{0} \nu \operatorname{Sg} \lambda_{i}\right) d \tau \\
x_{k}(\tau)=\alpha_{k}^{\sigma}(\tau) \int_{0}^{\tau} a_{\sigma}^{i}\left(S_{i}+f_{i} x_{4}-\delta_{0} \xi_{0} v \operatorname{Sg} \lambda_{i}\right) d \tau .
\end{gathered}
$$

Иіспользуя выражения $\mu_{i}(T)$ и $x_{k}(T)$ из $(2.11)$, получим для $\omega_{*}(0)$ формулы

$$
w_{v}(0)=\int_{0}^{T} a_{v}^{i}\left[h\left(f_{i} x_{4}-\delta_{0} \xi_{0} \nu S g \lambda_{i}\right)-\zeta_{i}\right] d \tau
$$

в которых $\lambda_{i}$ необходимо заменить согласно формулам $(1.17), x_{4}-$ согласно (2.9), $\psi_{4}-$ по формуле $(2.8)$, а $v$ определить как функцию $\mu_{i}$ на соответствующих многообразиях $h_{i}^{(a)}, h_{j}^{(b)}, h_{k}^{(c)}, h_{l}^{(d)}$. Тогда уравнение (2.12) определяет интегральные соотношения для постоянных величин $\varpi_{\nu}(0)$, после чего найти окончательное выражение для $v, \tau_{i}(\tau), \psi$, $\xi_{i}(\tau), x$ не представит собой трудностей. Тем самым будут определены значения $x_{i}(T)$. Относительно функции $U\left(R, i_{1}, i_{2}, \sigma_{1}, \sigma_{2}, \sigma_{3}\right)$ уместно предположить для орбитальных движений. при $\tau>T$, что она представима в виде

$$
U=\delta(t) U_{0}\left(\sigma_{1}, \sigma_{2}, \sigma_{3}\right)\left|U_{\mathrm{d}}\right| \simeq 1,
$$

где $\delta(T) \simeq \varepsilon, \delta(\tau)<\varepsilon$, при $T<\tau<T+\varepsilon, \delta(\tau)=0$ при $T+\varepsilon \leqslant \tau$,

так что, оставаясь в пределах нулевого приближения по $\varepsilon$, уместно считать систему $H$ помещенной в свободное пространство.

Будем считать на втором этапе $I_{10}=I_{20}+\varepsilon$, что справедливо для симметричных систем $H$. На втором этапе задача приводится к вопросу минимизации интеграла

$$
\begin{gathered}
\int_{7}^{T_{1}}\left\{v^{2}\left(\xi_{1}+\xi_{2}+\xi_{3}\right)+\delta_{0}^{2}\left[\left(p^{2}+q^{2}\right) \xi_{1}+\left(q^{2}+r^{2}\right) \xi_{2}+\left(p^{2}+r^{2}\right) \xi_{3}\right]-\right. \\
\left.-2 \delta_{0} v\left(p \xi_{1}+q \xi_{2}+r \xi_{3}\right)\right\} d \tau \quad\left(T_{1}=t_{2}-t_{0}\right) .
\end{gathered}
$$

Достаточно предположить, в силу неотрицательности $f^{(0)}$, что $\xi_{i}=0$ на $\tau$ из $\left[T, T_{1}\right]$, чтобы расход энергии при заданных относительно $x_{i}, \Omega$ граничных условиях на данном этапе оказался минимальным. Тогда система уравнений для объекта управления, учитывая уравнения (1.5) в 
предположении относительно уравнений (1.11), а также уравнений (1.6), будет следующая:

$$
\begin{aligned}
& \frac{d \omega_{1}}{d \tau}=-e \omega_{3} \omega_{2}+a v_{1} \\
& \frac{d \omega_{2}}{d \tau}=e \omega_{3} \omega_{1}+a v_{2} \\
& \frac{d \omega_{3}}{d \tau}=\frac{a}{c} v_{3} \\
& \frac{d \bar{k}}{d \tau}=[\bar{\Omega}, \bar{k}]-\bar{v} \\
& \frac{d \bar{\sigma}}{d \tau}=[\bar{\Omega}, \bar{\sigma}] \\
& \frac{d \psi}{d \tau}=\frac{\omega_{1} \sigma_{1}+\omega_{2} \sigma_{2}}{\sigma_{3}^{\prime}-1} .
\end{aligned}
$$

Здесь $e=1-I_{30} I_{10}^{-1}, a=I_{10}^{-1}, c=1-e, v_{i}=d_{1} k_{i} / d_{1} \tau, \bar{v}=\left(v_{1}, v_{2}, v_{3}\right)$. Используем $v_{i}$ для решения задачи о быстрейшем времени перехода системы $H$ из состояния $t_{0}$ в состояние $t_{1}$ или, исходя из метода обратной интеграции, не будем считать $t_{0}$ заданным.

Ввиду симметрии системы $H$ относительно оси $\mathrm{Oz}$ откажемся от требования быстродействия по $\varphi$ и, более того, от необходимости достижения последней в момент $T_{1}$ предписанного нацального значения $\varphi\left(T_{1}\right)$. Система уравнений для определения импульсов с учетом допущения и уравнений $(2.13)-(2.16)$ запишется так:

$$
\begin{aligned}
& \frac{d \psi_{1}}{d \tau}=-e \omega_{3} \psi_{2}+k_{3} \psi_{5}-k_{2} \psi_{6}+y_{0} \frac{\sigma_{1}}{1-\sigma_{3}^{2}}+z \frac{\sigma_{2}}{\sqrt{1-\sigma_{3}^{2}}} \\
& \frac{d \psi_{2}}{d \tau}=e \omega_{3} \psi_{1}+k_{1} \psi_{6}-k_{3} \psi_{4}+y_{0} \frac{\sigma_{2}}{1-\sigma_{3}^{2}}-z \frac{\sigma_{1}}{\sqrt{1-\sigma_{3}^{2}}} \\
& \frac{d \psi_{3}}{d \tau}=e\left(\omega_{2} \psi_{1}-\omega_{1} \psi_{2}\right)+k_{2} \psi_{4}-k_{1} \psi_{5} \\
& \frac{d \bar{x}}{d \tau}=[\bar{\Omega}, x] \\
& \bar{x}=\left(\psi_{4}, \psi_{5}, \psi_{6}\right) \\
& \frac{d y_{0}}{d \tau}=0, \quad y=y_{0} \\
& \frac{d z}{d \tau}=-y_{0} \frac{\sigma_{3}}{\left(1-\sigma_{3}^{2}\right)^{3 / 2}}\left(\omega_{1} \sigma_{1}+\omega_{2} \sigma_{2}\right) .
\end{aligned}
$$

Из условия максимума Л. Понтрягина функции $v_{i}$ следует определить в виде релейных управлений 


$$
\begin{aligned}
& v_{1}=k_{10} \operatorname{Sign}\left(a \psi_{1}-\psi_{4}\right) \\
& v_{2}=k_{10} \operatorname{Sign}\left(a \psi_{2}-\psi_{5}\right) \\
& v_{3}=k_{10} \operatorname{Sign}\left(a \psi_{3}-c \psi_{6}\right) .
\end{aligned}
$$

Фундаментальную систему решений уравнений (2.18) запишем в внде трех ортонормированных векторов $\overline{p_{j}}$; можно считать, что $\bar{\sigma}=a \bar{p}_{1}+b \bar{p}_{3}\left(a^{2}+b^{2}=1\right)$, для чего необходимо подобрать поворот на некоторый угол $\varphi_{0}$. Очевидно, что векторы $\bar{x}$, полный момент количества движения системы относительно точки $O-\bar{K}, \vec{v}$, решающий однородную систему уравнений для (2.14), можно определить линейными комбинациями векторов $\bar{p}_{i}$ с постоянными коэффициентами $x_{i}, K_{i}, v_{i}$. Тогда для $\bar{k}$ получим формулу

$$
\bar{k}=\left[v_{i 0}-\varphi_{i}(\tau)\right] \bar{p}_{i},
$$

где $v_{i 0}$, как проекции вектора $\bar{k}(T)$ на оси $O p_{i}$, известны из формул (2.11) и значений $a, b$ и $\varphi_{0}$, а функции $\varphi_{i}(\tau)$ суть

$$
\varphi_{i}(\tau)=\int_{\bar{T}}^{\bar{v}}\left(\bar{v}, \bar{p}_{i}\right) d \tau
$$

причем ввиду $\bar{k}\left(t_{0}\right)=0$ имеем

$$
v_{i 0}=\varphi_{i}\left(T_{1}\right)
$$

Рассмотрим векторное равенство

$$
\left[K_{i}-v_{i 0}+\varphi_{i}(\tau)\right] \overline{p_{i}}=\bar{G}(\vec{\omega})
$$

$\left(\bar{G}(\bar{\omega})\right.$ имеет на главные оси проекции $\left.I_{1} \omega_{1}, I_{2} \omega_{2}, I_{3} \omega_{3}\right)$, выражающее по теореме Резаля постоянство вектора $\bar{K}$ в неподвижном пространстве для принятых обозначений. Из равенства (2.25) для $\omega_{1}, \omega_{2}, \omega_{3}$ имеем выражения

$$
\begin{aligned}
\omega_{1} & =a\left[m_{1} p_{11}+m_{2} p_{21}+m_{3} p_{31}\right] \\
\omega_{2} & =a\left[m_{1} p_{12}+m_{2} p_{22}+m_{3} p_{32}\right] \\
c \omega_{3} & =a\left[m_{1} p_{13}+m_{2} p_{23}+m_{3} p_{33}\right] \\
& \left(m_{i}=K_{i}-v_{i 0}+\varphi_{i}\right) .
\end{aligned}
$$

С другой стороны, из первых двух уравнений системы (2.13) и (2.17) получим для $\omega_{1}+i \omega_{2}, \psi_{1}+i \psi_{2}$ выражения:

$$
\omega_{1}+i \omega_{2}=\left[\omega_{1}(T)+i \omega_{2}(T)+\left.a\right|_{i}\left(v_{1}+i v_{2}\right) \exp (-\omega(\tau)) d \tau\right] \exp \omega(\tau)
$$

$\psi_{1}+i \psi_{2}=\left[\psi_{1}(T)+i \psi_{2}(T)+\int_{T}^{\tau}\left(N_{1}+i N_{2}\right) \exp (-w(\tau)) d \tau\right] \exp w(\tau)$ 
где обозначень:

$$
\begin{gathered}
\omega(\tau)=i e \int_{i} \omega_{3} d \tau, \quad N_{1}=n_{i} p_{i 1}+y_{0} \frac{\sigma_{1}}{1-\sigma_{3}^{2}}+z \frac{\sigma_{2}}{\sqrt{1-\sigma_{3}^{2}}} \\
N_{2}=n_{i} p_{i 2}+y_{0} \frac{\sigma_{2}}{1-\sigma_{3}^{2}}-z \frac{\sigma_{1}}{\sqrt{1-\sigma_{3}^{2}}}, \quad\left[\bar{x}, \overline{v_{0}}-\bar{\varphi}(\tau)\right]=\bar{n}=\left(n_{1}, n_{2}, n_{3}\right) \\
\bar{x}=\left(x_{1}, x_{2}, x_{3}\right), \quad \bar{\varphi}=\left(\varphi_{1}, \varphi_{2}, \varphi_{3}\right), \quad \bar{v}=\left(v_{10}, v_{20}, v_{30}\right) .
\end{gathered}
$$

Из третьего уравнения системы (2.13) имеем для $\omega_{3}$ выражение

$$
\omega_{3}(\tau)=\omega_{3}(T)+\frac{a}{c} \int_{i} v_{3} d \tau
$$

которое необходимо подставить в строки (2.27) и (2.28). Исключая из формул $(2.26),(2.27),(2.28)$ и (2.29) переменные $\omega_{1}, \omega_{2}, \omega_{3}$, нетрудно получить для $p_{i j}$ три интегральных уравнения, дополняюших шесть условий ортонормированности этих функций. Подставляя решения этих уравнений $p_{i j}=p_{i j}^{0}\left(v_{1}, v_{2}, v_{3}, \tau\right)$ в формулы $(2.26)$, получим выражения для $\omega_{i}=\omega_{i}^{0}\left(v_{1}, v_{2}, v_{3}, \tau\right)$. Аналогично получим формулы для $k_{i}^{0}\left(v_{1}, v_{2}, v_{3}, \tau\right), \quad x_{i}^{0}\left(v_{1}, v_{2}, v_{3}, \tau\right), \quad \sigma^{0}\left(v_{1}, v_{2}, v_{3}, \tau\right)$, a $\psi=\psi^{0}\left(v_{1}, v_{2}, v_{3}, \tau\right)$ и $z=z^{0}\left(v_{1}, v_{2}, v_{3}, \tau\right)$, интегрированием из (2.16) и (2.20). Используя эти величины и принятые обозначения в равенстве $(2.28)$, получим $\psi_{1}=\psi_{1}^{(0)}\left(v_{1}, v_{2}, v_{3}, \tau\right)$ и $\psi_{2}=\psi_{2}^{(0)}\left(v_{1}, v_{2}, v_{3}, \tau\right)$. После этого значение $\psi_{3}=\psi_{3}^{(0)}\left(v_{1}, v_{2}, v_{3}, \tau\right)$ определяется интегрированием третьего уравнения (2.17). После замены в равенствах $(2.21)$ величин $\psi_{l}(l=\overline{1,6})$ на решения $\psi_{l}^{0}\left(v_{1}, v_{2}, v_{3}, \tau\right)$ получим уравнения для определения $v_{i}=v_{i}^{0}(\tau)$, что в принципе позволит считать все фазовые координаты известными функциями времени. Параметры $\psi_{i}(T), x_{i}, y_{0}, z(T) \quad(i=\overline{1,3})$ соответствуют восьми граничным условиям на фазовые координаты - $\omega_{i}\left(T_{1}\right), k_{i}\left(T_{1}\right)$, $\sigma_{3}\left(T_{1}\right)$ и $\psi\left(T_{1}\right)$. Отметим, что сходная задача о наибыстрейшем торможении симметричного тела полностью решена в работе [ $\left.{ }^{4}\right]$.

\section{Л ИТЕРАТ У Р А}

1. Р у м ян це в В. В., ПММ, 25, вып. 1, 9-16 (1961); ПММ, 25, вып. 5, 778-784 (1961)

2. А ми н ов М. Ш., Тр. Қазанск. авиационного ин-та. Матем. и механика, 48, 1-47 (1959).

3. Понтрягин Л. С., Болтянский В. Г., Гамкрелидзе Р. В., Мищен к о Е. Ф., Математичєская теория оптимальных процессов. М., 1961, стр. $68-80$.

4. С мольников Б. А., ПММ, 28, вып. 4, 725-734 (1964).

Ннститут кибернетики Академии наук Эстонской ССР
Поступила в редакцию 17/XII 1965 
I. KEIS

\section{GUROSTAATILISE LENNUAPARAADI LIIKUMISEST UMBER KINNISPUNKTI MINIMAALSE ENERGIAKULUGA}

Artiklis vaadeldakse gürostaatilise lennuaparaadi viimist algasendist teatud punkti ning aparaadi edasist kindla orientatsiooniga manööverdamist minimaalse energia. kuluga.

Ulesanne lahendatakse Pontrjagini maksimumprintsiibi abil kahes etapis. Esmalt vaadeldakse liikumist kuni manöövri alguseni. Sel juhul on liikumisvőrranditeks kolm integraalvōrrandit ëheksa funktsiooniga, mis rahuldavad veel kuut ortonormeerimise tingimust. Teisel etapil vaadeldakse lennuaparaadi liikumist manöövri lōpuni.

I. KEIS

\section{ABOUT THE ENERGY MINIMIZATION PROBLEM OF A GYROSTAT MOTION AROUND A FIXED POINT}

The problem of a gyrostat motion transfer from the initial stage to the predicted orientation manoeuvre economizing the energy during its flight is considered in the paper. Pontryagin's method was used in the two parts of its solution - the first one, dealing with placing the gyrostat into the prescribed point at the manoeuvre, reduces itself to the problem of the existence of solvents of several integral equations, while the second one - the pure manoeuvre, is solved completely. 